\title{
Automatic Identification of Dataset Names in Scholarly Articles of Various Disciplines
}

\author{
Daisuke Ikeda *, Kota Nagamizo *, Yuta Taniguchi *
}

\begin{abstract}
Although the number of freely accessible scholarly articles is increasing, it is difficult for non-experts to understand them since they are written for experts and require background knowledge. Our big goal is to facilitate open innovation based on scholarly articles, developing methods to automatically extract essential elements in them. Once we could understand articles, they would be primary resources for institutional research. To this end, this paper is devoted to developing automatic identification of datasets in articles. Because a dictionary of datasets is necessary for evaluation, existing methods focused on some specific discipline. To achieve applicability to any disciplines, a machine learning approach with huge amounts of papers is adopted. Treating papers in multi-disciplines, the authors are not familiar with all dataset names in them. Therefore we quantitatively evaluate experimental results with precision@N, which does not require to know all the datasets in the papers, and qualitatively check if candidate tokens are dataset names or not using a GUI tool we have developed. Experimental results show precision@N is 0.450 and nDCG is 0.458 . However, outputs include names of methods and software. It is an importantfuture work to remove these noise tokens.
\end{abstract}

Keywords: scholarly repository, dataset name identification, vector representation, precision@N

\section{Introduction}

Scholarly repositories are databases of scholarly articles, and the number of scholarly repositories is drastically increasing. In fact, there exist more than two million full texts on about 800 institutional repositories in Japan, as of March, 2019 [1]. In addition to institutional repositories, there also exist many disciplinary repositories, mainly maintained by scholarly communities, such as arXiv [2], PubMed [3], RePEC [4], and SSRN [5].

Thanks to recent wide-spread of scholarly repositories, we can freely access to a huge number of scholarly articles on them. Since scholarly papers contain some knowledge about a discipline, we can expect to facilitate open innovation, reusing these papers and combining knowledge among different disciplines.

\footnotetext{
* Kyushu University, Fukuoka, Japan
} 
However, it is difficult in general for general public or even researchers in other disciplines to understandscholarly articles since they are basically written for domain experts. Nevertheless, the authors believe that we can develop automatic ways to give scholarly papers a structure because scholarly papers have some format, which make domain experts read papers in their domain quickly.

The big goal of our research is to facilitate open innovation through freely available scholarly papers, developing methods to extract essential elements of them, such as materials and methods. Once we obtain such elements, combining ideas, methods, or materials in different disciplines can lead to open innovation.

From the viewpoint of institutional research, data on institutional repositories, such as the number of published papers or the number of accesses to them, can be a good resource to evaluate researches in institutions because an institutional repository is managed by an institution, such as a university, to provide scholarly contents created by members of the institution. For example, access log data of an institutional repository shows that many non-researches have accessed to contents on an institutional repository [6]. Such a fact reveals that scholarly papers, which are usually considered for researchers, are appealing to non-researchers.

Institutional research is basically conducted based on statistical data, or data collected with original systems [7],[8]. In addition to statistical data on repositories, the authors believe that contents on them, scholarly papers, would be great resources for institutional research once we could understandscholarly articles automatically because scholarly papers are direct outcomes from research. With such resources, for example, a research administration office of a $\mathrm{n}$ institutionc ould facilitate collaborative research projects $\mathrm{w}$ ithin the same institution.

Toward the goal, the authors consider the following task: given a set of scholarly articles, automatically identify dataset names in them. The authors chose this task because it seems easier, compared to identify any concepts in text data, and it will be importantfor rich indexes at data repositories to identify dataset names [9].

In the field of text mining or natural language processing, similar tasks have been studied as information extraction or named entity extraction (see Section 2), and our task could be considered as a subtask of them. Methods based on these existing tasks assume that given text data is written in one language and there exist some common patterns in the text data. However, now we are considering scholarly articles of various disciplines, we can not assume some common patterns because writing styles or vocabularies differ depending on disciplines even if papers are written in one language, English.

Some researches about datasets have emerged recently, such as dataset name extraction, discovery of links to datasets, and dataset search engines [9][10][11][12]. Existing methods about datasets have focused on a small set of papers in some specific discipline. But, when we want to use identified dataset names for o pen innovation or c ollaborative research, it is crucial for such methods to be applicable to many disciplines. Therefore the authors consider dataset names in various disciplines and use papers collected by CORE [13], which collects papers on institutional repositories and provides search APIs, dumpfiles, and search facility for collected papers [14].

Existing methods for dataset name identification use two steps of candidate-generation and similarity check. For example, in [12], tokens of capital letters are extracted as candidates for dataset names and then they are removed if contained in a standard English dictionary. Next candidate tokens are extracted as dataset names if they have high similarities with word "dataset". The limitation on the candidate-generation step is so strict that 
there are unextracted dataset names, such as ACE, which is a dataset obtained with satellites by NASA, while the similarity check is based on a loose measure since they have used a general search engine to measure similarities. The authors use a looser pattern to extract candidate tokens while more strict similarity check using vector representation of words, word2vec [15][16].

Because we treat papers in multi-disciplines, including those the authors are not familiar with, it is challenging how to evaluate experimental results. To solve it, the authors quantitatively evaluate experimental results with precision@N and nDCG, where precision@N is a popular measure for information retrieval systems [17] and nDCG (normalized Discounted Cumulative Gain) is for recommendation systems [18]. Both measures can be calculated by the top $\mathrm{N}$ list of outputs. To check if candidate tokens of the top $\mathrm{N}$ list are dataset names or not, the authors use a GUI tool they have developed. The GUI tool is developed with standard Web technologies, such as CSS, you can use it on a Web browser.

Checked with the top 20 of high similarity values, the result shows that precision@N is 0.450 and $\mathrm{nDCG}$ is 0.458 . These figures are lower, compared to existing research. This is mainly due to our input articles are not restricted to one discipline.

This paper is an extended version of our conference paper [19], where some major differences include (1) a larger dataset of $330 \mathrm{~GB}$ with $9.8 \mathrm{M}$ full text papers is used, compared to one of $102 \mathrm{~GB}$ with $4 \mathrm{M}$ papers; (2) a larger subset of papers of $100 \mathrm{~GB}$ full text papers is used to construct a model of word2vec, compared to 3GB; (3) a much more looser pattern is adopted to find candidate tokens; (4) description of quality for metadata of papers obtained from scholarly repositories is added; (5) for evaluation, nDCG is also used, in addition to precision@N; and (6) compared to the previous result 0.350 of precision@N, we obtained 0.450 due to a larger set of scholarly articles.

\section{Related Work}

We can consider identification of dataset names as common pattern extraction. In other words, the authors adopt an unsuprvised approach, instead of supervised ones like [20]. So, first, we explain information extraction from the Web and named entity extraction as common pattern extraction, and then related work on dataset name identification.

\subsection{Common Pattern Extraction}

Finding common patterns is a central task of text mining and many tasks to find common pattern have been studied, depending on properties of inputtexts. In case of semi-structured data, news articles are extracted from Web pages at a news site after identifying common templates of the site as common patterns [21][22]. In case of natural language texts, named entity extraction have been extensively studied. In this task, the person names, organizations, locations, etc., are called named entity.

Named entities are common expressions to one language, and dataset names are also common expression to one discipline. In this sense, the former task treats expressions distributed in a wider area while the latter ones distributed in a narrow area. This means that, in case of dataset name identification, there are many local expressions depending on disciplines, and thus it is more challenging. In case of information extraction from the Web, although each site has its own template and there are a lot of local templates, these templates are basically written in artificial languages, such as HTML, which are suitable to automatic processing. 


\subsection{Dataset Name Extraction}

Assuming one fixed dataset, Ikeda and Seguchi developed a classifier based on support vector machines, which classifies if a given paper uses the dataset or not [9]. They used words appeared near figures, tables, and acknowledgment as features to train the classifier. It can check if one dataset is used in a paper or not. But, this method fixed one dataset name and thus is not applicable to our task.

In [10], Ghavimi et al. tried to identify links to datasets of social science, but not to extract dataset names.

In [12], Singhal and Srivastava extracted tokens of capital letters from predefined sections, such as "Experiments" and "Results". The authors think that this assumption holds because the authors use papers of only one discipline. Using pattern matching, extracted tokens were deleted if they appear in standard English dictionaries. For each candidate of extracted tokens, Singhal and Srivastava calculated the similarity with "dataset", based on NGD (Normalized Google Distance) [23]. To calculate similarities based on NGD, Singhal and Srivastava used a standard search engine. Therefore, costly preprocessing or training examples are not necessary. However, since candidate tokens must consist of capital letters and not be standard English words, there must exist many dataset names not extracted as candidates by their approach.

In addition to that, we can not expect that extracted dataset names would be useful for open innovation because Singhal and Srivastava evaluated using only 50 papers. Even in [24] by the same authors, only 400 papers were used to evaluate their proposed method.

\section{Materials and Methods}

\subsection{Methods}

Like the approach in [12], we also first generate candidate tokens and then check if, for each candidate, the similarity between words, such as "dataset" and "database".

We use two settings for candidate generation: strict patterns and loose ones, where, in the former setting, a candidate token can contain at least one lower-case letter and some symbols, including "-", "+", and in the latter case, a candidate token must contain at least one upper-case letter. It is noteworthy that this strict pattern generation is less strict than the candidate generation in [12], where only upper-case letters are allowed in a candidate token.

Let $A B C D$ be such a token. We assume that a token is an abbreviation of some proper name and the abbreviation, that is the token, first appears near the proper name. Therefore, we use the following regular expression to find its proper name in case of the token: “a.* b.* c.* d.*”. Using a window size as a parameter for being neighbor, we search a proper name in the window size around candidate token. In this experiment, we set 20 for the window size, that is we search 20 tokens for proper names before or after each candidate token.

For each candidate token, we calculate the similarity value of the token with one of the following words using a trained model of word2vec: "dataset", "datasets", "database", or "databases", where cosine similarity is used to measure similarity between two tokens. These words are chosen after some preliminary experiments. Given some threshold for similarity, similar tokens are extracted as dataset names. 
Table 1: The numbers of papers with some values and no values for each metadata type

\begin{tabular}{lrr} 
key & \#valid & \#null \\
\hline abstract & 11229482 & 5676916 \\
authors & 14617965 & 2288433 \\
contributors & 33329883 & 13573415 \\
coreId & 16906398 & 0 \\
datePublished & 14791413 & 2114985 \\
doi & 2574246 & 14332152 \\
downloadUrl & 2087088 & 14819310 \\
enrichments & 16906398 & 0 \\
fullTextIdentifier & 5262691 & 11643707 \\
identifiers & 656535 & 16249863 \\
journals & 2443697 & 14462701 \\
language & 644301 & 16262097 \\
oai & 15277463 & 16288935 \\
pdfHashValue & 2076865 & 14829533 \\
publisher & 10137080 & 6769318 \\
raeRecordXml & 16905316 & 1082 \\
relations & 7424331 & 9482067 \\
subjects & 15669487 & 346911 \\
title & 16714395 & 192003 \\
topics & 11595442 & 5310956 \\
year & 14717716 & 2188682
\end{tabular}

\subsection{Data}

We use the dump file provided by CORE [13], as of March, 2018. According to the Web page for the dataset, the size of the latest dump file in zip format is about 330GB, containing more than 9.8 million full text papers. After removing some papers which can not be read successfully, the total size is $576 \mathrm{~GB}$ of 7.7 million full text papers.

CORE collects papers on institutional repositories and thus we can obtain papers in various disciplines, unlike disciplinary repositories. However, in general, the quality of metadata on institutional repositories is not good. To see this, the authors checked if a field of metadata has a value or not, using about 16.9 million papers sampled from 123 million metadata-only papers in the dump file.

Table 1 shows a metadata type (key), the number of papers which contain some values (\#valid) and no values for the metadata type (\#null). For example, 11,229,482 papers have some values in "abstract" but 5,676,916 papers do not have in this field. The authors believe that "journals" and "topics" would be useful to detect a discipline of the paper, but most of the papers do not have valid values in these fields.

As described before, many papers do not have valid values for "language" field. Therefore, as preprocessing, first the authors selected papers written in English, using langdetect module of Python. For a document $d$, function detect langs $(d)$ in the module returns a list of probabilities of detected languages. For example, if we obtaine the following list:

[sw:0.7142823673474135, lt:0.2857133611277489],

then we consider that the paper is written in Swahili in about $71 \%$ and in Lithuanian in 
Table 2: Top 20 frequent tokens from the papers used in this paper

\begin{tabular}{rrl|rrl} 
rank & frequency & token & rank & frequency & token \\
\hline 1 & $301,694,228$ & the & 11 & $32,762,908$ & as \\
2 & $192,812,517$ & of & 12 & $28,856,878$ & by \\
3 & $148,652,763$ & and & 13 & $26,770,867$ & on \\
4 & $116,120,727$ & in & 14 & $26,428,859$ & this \\
5 & $101,235,862$ & to & 15 & $26,165,272$ & was \\
6 & $78,336,746$ & a & 16 & $25,276,836$ & are \\
7 & $52,734,608$ & is & 17 & $24,695,921$ & be \\
8 & $49,865,713$ & for & 18 & $22,296,880$ & from \\
9 & $40,890,890$ & that & 19 & $20,089,105$ & were \\
10 & $36,432,055$ & with & 20 & $19,473,401$ & at
\end{tabular}

Table 3: Top 20 frequent tokens from the smaller set of papers we used before

\begin{tabular}{rrl|crl} 
rank & frequency & token & rank & frequency & token \\
\hline 1 & $1,421,239$ & the & 11 & 179,747 & $\mathrm{~s}$ \\
2 & $1,033,674$ & of & 12 & 165,337 & by \\
3 & 805,680 & and & 13 & 155,649 & as \\
4 & 609,737 & in & 14 & 145,085 & on \\
5 & 545,384 & a & 15 & 140,174 & $\mathrm{e}$ \\
6 & 535,831 & to & 16 & 134,784 & be \\
7 & 300,485 & for & 17 & 133,660 & this \\
8 & 239,157 & is & 18 & 130,671 & $\mathrm{n}$ \\
9 & 200,136 & that & 19 & 129,517 & or \\
10 & 193,121 & with & 20 & 128,017 & was
\end{tabular}

about $29 \%$. We choose a document if the probability for English is equal to or greater than $90 \%$, that is en $\geq 0.9$.

Next the authors removed the following symbols:

[]\{\}()$=\mid-\langle>: ; "$ ' .,

After removing these symbols, they obtained tokens by separating with space. Then they converted digits into " 0 ".

Next, for each token, the authors translated the first capital letter of the token into the corresponding lower-case letter if the succeeding letters of the tokens are lower case. For example, "SaaS" is treated as it is while "This" and "We" are translated into "this" and "we", respectively.

Table 2 shows the top 20 of frequent tokens after the preprocessing, where each line shows the rank of a token, its frequency, and the token. In this list, we find many stop words, such as "the" and "of".

In Table 3, for comparison, the authors show the top 20 frequent words from the smaller set of papers we used in [19]. We see similar words in both lists but frequencies are much smaller than those in the list of Table 2.

The file size of papers after preprocessing is about 298GB, which is still too large to train a word2vec model. So we use 100GB tokens for training, which corresponds to 505,280 papers. 


\subsection{Setting}

Dell Precision Tower 7910 with 32 Intel Xeon $(2.10 \mathrm{GHz})$ as CPUs and $125.8 \mathrm{GiB}$ main memory, running Ubuntu18.04LTS, was used for experiment.

Programs were written in Python3.6.9, using Word2Vec module in gensim, a natural language processing library. As training parameters, skip-gram model was used, where the dimension size was 100 , the number of negative sampling 5 , the window size 10 , infrequent words whose frequencies are equal to or less than 4 were removed, and we used 32 parallel processing.

\section{Experiments}

\subsection{Evaluation}

Unlike existing methods, the authors do not assume some specific discipline and there do not exist any datasets or dictionaries of dataset names, as far as the authorsknow. Therefore it is challenging how to evaluate results. In particular, our target is abbreviations, consisting of several letters. Therefore, it is difficult to check if given abbreviations are dataset names or not unless we are familiar with them in advance. Although we can make full use of corresponding proper names for abbreviations, we have to check the context in which some abbreviation is used.

For this purpose, the authors developed a GUI tool, which shows, given a query of regular expression, matched patterns and their neighbors in original texts (see Figure 1). In this example, the given query is " $\backslash \mathrm{WCVD} \backslash \mathrm{W}$ ", which means "CVD" surrounded with word-delimiters " $\backslash W$ ", where " $\backslash W$ " is a special symbol of regular expression. It matches, for example, "(CVD)" and “-CVD" ", but not "ECVD)" because "E" is nota word-delimiter

In information retrieval, precision and recall are typical measures to evaluate the whole output of an information retrieval system. Precision (resp. recall) is the ratio of correctly retrieved tokens to retrieved token (resp. relevant tokens, that is dataset names) [17]. However, to calculate these measures, we need to know all dataset names in advance. The above GUI tool can be used to evaluate each tokens, but not for the whole output of a dataset nameidentification system. Therefore, instead of precision and recall, we use precision@N, which is used with a ranked list and defined as precision at top $\mathrm{N}$ tokens. In this case, we can check if a token in the top $\mathrm{N}$ tokens is a dataset name or not.

In addition to precision@N, we also calculate nDCG, normalized Discounted Cumulative Gain [18], which is used to evaluate ranked outputs, such as recommendation. In particular, nDCG is often used for outputs of items $w_{i}$ with relevance score eval $\left(w_{i}\right)$. Using eval $\left(w_{i}\right)$, DCG for top $k$ ranking is defined by

$$
\mathrm{DCG}=\sum_{i}^{k} \operatorname{eval}\left(w_{i}\right) / \log _{2}(i+1),
$$

while the ideal DCG, denoted by $\mathrm{DCG}_{I}$, is defined by

$$
\mathrm{DCG}_{I}=\sum_{i} 1 / \log _{2}(i+1) .
$$

\footnotetext{
${ }^{1}$ Note that a space follows "CVD".
} 
C 亩 (1) file:/lI app.html … 约

\begin{tabular}{|c|}
\hline $\mid$ WCVDIW \\
\hline Showing 100 matches
\end{tabular}

rown on iridium (Ir) by direct current plasma chemical vapor deposition(CVD). with plasma enhanced chemical vapor deposition (PECVD) and thermal CVD. PECVD-grown CNTs contained the fragmented catalytic nanoparticles Subsequent therma CVD resulted in the secondary growth of CNTs on these catalytic nanoparti .n these catalytic nanoparticles. The CNTs grown by PECVD and therma CVD showed the turn-on electric fields of about 4.5 and $3.5 \mathrm{~V} / \mathrm{mum}$ for elec very high-frequency plasma-enhanced chemical vapor deposition (VHF-CVD) technique, were investigated. It was concluded that the VHF-CVD tech - (VHF-CVD) technique, were investigated. It was concluded that the VH-CVD technique with a high hydrogen dilution of silane and germane is suitab $d$ to the high density of hydrogen radicals, which are proper to the VH-CVD technique. -netration of hydrogen atoms into the film. In device fabrication, the VH-CVD techniqu on rate for the a-SiGe:H photovoltaic layer than those in conventional R-CVD methods ith parylene was achieved by a low-pressure chemical vapor deposition(CVD) techniq en utilized in practical applications. Although chemical vapor deposition(CVD) provides $r$ deposition (CVD) provides high quality coatings, the deposition rate $\circ$ CVD could ha - have been too small for TBCs. We have recently developed a new lase CVD process : ursors. An Nd:YAG laser with a high power of $250 \mathrm{~W}$ was introduced in CVD chamber oxides such as $\mathrm{Y} 2 \mathrm{O} 3, \mathrm{Al} 2 \mathrm{O} 3$ and TiO2 films were also prepared by lase CVD at high d where synthesized by plasma enhanced chemical vapor deposition (PE-CVD) using th $s$ problem, we developed a practical large-radius neutral beam enhance CVD process : ition, assisted with the plasma generated by radio frequency waves RF CVD (13,56M! $n$ films were grown by plasma-enhanced chemical vapor deposition (PE-CVD) using irc ere produced using high cost technologies: Chemical Vapor Deposition(CVD), Physica hydrogenated amorphous silicon germanium chemical vapor deposition with a cherical Shima, M., Isomura, M., Wak JAPANESE JOURNAL OF APPLIED PHYSICS PART 1-REGULAR $\mathrm{nU} \mathrm{m} / \mathrm{h}$ PAPERS SHORT NOTES \& REVIEW PAPERS, 42(12), 7198-7204 a whol DEC, 2003
$10.1143 /$ JJAP.42.7198

solar cell, amorphous silicon germanium, very high frequency S) and (VHF), plasma-enhanced chemical vapor deposition, (PE-CVD), any $p$ hydrogen dilution, light-induced degradation, hydrogen bonding configuration

Ways of improving the deposition rate of hydrogenated amorphous
silicon germanium (a-Sige:H) while maintaining its film quality,

using the very high-frequency plasma-enhanced chemical vapor
using ind the
deposition (VHF-CVD technique, were investigated. It was
concluded that the VHF-CVD technique with a high hydrogen

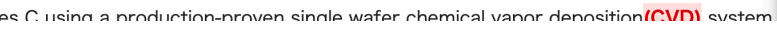

Figure 1: Screenshot of the developed GUI tool, given " $\backslash W C V D \backslash W$ ", which means CVD surrounded with word-delimiters $(\backslash W)$, as a regular expression. The tool shows matched results.

With DCG and $\mathrm{DCG}_{I}$, $\mathrm{nDCG}$ is defined as follows:

$$
n D C G=\frac{\mathrm{DCG}}{\mathrm{DCG}_{I}} .
$$

A value of $\mathrm{nDCG}$ is between 0.0 to 1.0 , and reaches to the maximail value if the ranked outputis equal to the ideal ranking. Compared to precision@N, a value of $\mathrm{nDCG}$ is sensitive to the ranking of outputs.

In our experiments, we set $k=20$ and, for the $i$ th element $w_{i}$ in the top $k$ of an output, we use

$$
\operatorname{eval}\left(w_{i}\right)= \begin{cases}1 & w_{i} \text { is a dataset name } \\ 0 & \text { otherwise }\end{cases}
$$

\subsection{Results}

The authors created two models of word2vec, using $3 \mathrm{~GB}$ and 100GB text data. To show some differences of the data size, first we show the result from $3 \mathrm{~GB} d$ ata. In case of $3 \mathrm{~GB}$ data, only strict pattern generation is used, where a pattern may contain one lower-case letter.

Table 4 shows the top 20 tokens of high similarity values, where the first column is for token, the second one for its similarity value, the third one for our judgment, where 1 denotes the authorsfound it as a dataset name. From original texts of the GUI tool, the authors 
Table 4: Top 20 tokens which have highest similarities to "dataset", "datasets", "database", or "databases", their similarities, and our judgment, calculated using the strict pattern generation and the model tained with $3 \mathrm{~GB}$ text data.

\begin{tabular}{lrr} 
token & similarity & judgment \\
\hline MSLA & 0.7069758 & 1 \\
CBIR & 0.6979505 & 0 \\
PCA & 0.6379499 & 0 \\
VGAM & 0.6051862 & 0 \\
KDHS & 0.5983720 & 1 \\
\hline BLASTP & 0.5928094 & 0 \\
GIS & 0.5722876 & 0 \\
ORES & 0.5683997 & 0 \\
SESA & 0.5669162 & 0 \\
SCFD & 0.5655370 & 0 \\
\hline CYGD & 0.5646238 & 1 \\
CoPS & 0.5627788 & 0 \\
EBI & 0.5610322 & 1 \\
IASCF & 0.5582382 & 0 \\
OPN & 0.5544391 & 0 \\
\hline OALD & 0.5532236 & 1 \\
KIMR & 0.5480386 & 1 \\
NVL & 0.5462620 & 0 \\
NMRFS & 0.5445106 & 1 \\
LARALL & 0.5428956 & 0 \\
\hline
\end{tabular}

judged 7 out of 20 are dataset names and thus precision@N is 0.350 . However, methods or models, like PCA and VGAM, are also included. We also find names for organization.

In this table, the authors only see tokens with upper-case letters although one lowercase letter is allowed in tokens. Our method allows one lower-case letter while the existing method allowed only upper-case letters in tokens. However, this result means this relaxation might not be enough.

From this table, we find that these tokens have various values of similarity, ranging from 0.7 to 0.54 . This fact means that tokens at lower ranks are not so close to "database" or "dataset". Therefore, we can not conclude it has high quality with this model.

Next we move to the result from the larger size of text data. Table 5 shows the top 20 tokens which have highest similarity values, where these similarity values are calculated using the strict pattern generation and the model tained with 100GB text data. In this list, we see one token, "iHMS", has one lower-case letter in it.

Thanks to the size of text data, we also find that tokens in this list have much higher similarity values, ranging from 0.78 to 0.72 , compared to Table 4 .

The authors judged 9 out of 20 are dataset names, that is precision@N is 0.450 and $\mathrm{nDCG}$ is 0.505 .

Next let us see effect of pattern generation condition. Table 6 shows the top 20 tokens which have highest similarity values, where these similarity values are calculated using the loose pattern generation and the model tained with 100GB text data. In this condition of patterngeneration, if at least one upper-case letter is included in a token, we treat the token 
Table 5: Top 20 tokens which have highest similarity values to "dataset", "datasets", "database", or "databases", their similarity values, and our judgment, calculated using the strict pattern generation and the model tained with $100 \mathrm{~GB}$ text data.

\begin{tabular}{lrr} 
token & similarity & judgment \\
\hline GPKB & 0.789373457 & 1 \\
GEOGLE & 0.770034313 & 0 \\
iHMS & 0.751895964 & 1 \\
OBDA & 0.751499474 & 0 \\
PHIDIAS & 0.749419689 & 1 \\
\hline REGSTATTOOLS & 0.747586489 & 0 \\
GPMDB & 0.743063569 & 1 \\
GMQL & 0.742300868 & 1 \\
POINeT & 0.742185473 & 0 \\
KUPKB & 0.741823316 & 1 \\
\hline MITAB & 0.738892317 & 0 \\
VIRTUAL2D & 0.738280594 & 0 \\
DMRR & 0.735069335 & 0 \\
HCDN & 0.734469473 & 1 \\
SB AL & 0.733836412 & 0 \\
\hline BICOMB & 0.728748083 & 0 \\
SABINET & 0.727956653 & 1 \\
SQIV & 0.726967931 & 0 \\
COPASAAR & 0.725873709 & 1 \\
CYTOSCAPE & 0.725675702 & 0 \\
\hline
\end{tabular}

as a candidate for dataset names. We can see that this relaxing leads to many tokens with many lower-case letters, such as "Pro-Cite" and "ProteinQuest".

The authors also judged 9 out of 20 are dataset names, thus precision@N is 0.450 , equal to that from Table 5. However, ranking is different between Table 5 and Table 6 , and thus $\mathrm{nDCG}$ is 0.458 , which is a little smaller than $\mathrm{nDCG}$ of Table 5. In general, widening candidate generation pattern may increase the number of non-datasetnames. In this sense, this result is notbad since values for precision@N are the same between Table 5 and Table 6.

\subsection{Discussion}

In the previous section, we can find many dataset names in Table 5 and Table 6 . However, more than a half of the top 20 tokens are judged as non-dataset names. Therefore, it is important to remove such non-dataset names. Using word2vec model, we can find words sharing similar contexts. In other words, if two words appear in similar contexts, they are treated as similar. Since our approach is heavily depends on the trained word2vec model, the extracted tokens must share similar contexts. The results include many names of methods and models, such as PCA, because these names and dataset names are often used in similar contexts. Thus, just using similarities between some specific words, such as "database", is not enough to extract only dataset names.

It is well known that word vectors obtained by word2vec have additive compositionality [16]. So we can use plus or minus operations to word vectors. For example, the 
Table 6: Top 20 tokens which have highest similarity values to "dataset", "datasets", "database", or "databases", their similarity values, and our judgment, calculated using the loose pattern generation and the model tained with $100 \mathrm{~GB}$ text data.

\begin{tabular}{lrr} 
token & similarity & judgment \\
\hline GPKB & 0.789373457 & 1 \\
Pro-Cite & 0.783878148 & 0 \\
SynapticDB & 0.782725871 & 0 \\
ArrayWiki & 0.77789259 & 0 \\
GEOGLE & 0.770034313 & 0 \\
\hline GenderMedDB & 0.765523553 & 1 \\
CrystalEye & 0.764046669 & 1 \\
VarElect & 0.760374784 & 0 \\
CasJobs & 0.760158062 & 0 \\
ProteinQuest & 0.759543657 & 0 \\
\hline JAX-CKB & 0.756258845 & 1 \\
ISI & 0.755305111 & 1 \\
exRNA & 0.755060971 & 1 \\
Web-accessible & 0.752642691 & 0 \\
iHMS & 0.751895964 & 1 \\
\hline NLM & 0.751669407 & 1 \\
OBDA & 0.751499474 & 0 \\
MetaPathways & 0.750950694 & 0 \\
rnaSeqMap & 0.749927461 & 0 \\
PHIDIAS & 0.749419689 & 1 \\
\hline
\end{tabular}

following equation holds:

$$
\text { "King" - "Man" + "Woman" = "Queen" }
$$

It it noteworthy that we did not find "Queen" from only "King", but find it from "King" with "Man" and "Woman", where "Man" and "Woman" play an role to fix a context of "King" and "Queen". In this sense, the authors believe that this property can improve results of our method. It is an importantfuture work to find good operations to remove methods, models, or organizations from similar tokens to "database" or "dataset".

To this end, we calculated similarity values and distances between non-dataset names and dataset ones. Figure 2 shows two graphs for cosine similarity. The left-hand side figure shows values of cosine similarity between vectors $\boldsymbol{v}_{0}$ of some tokens obtained by a trained word2vec model. The right-handside figure also shows values of cosine similarity, however target vectors are different from the left-hand side one, where the similarity is calculated between vectors $\boldsymbol{v}$ of the same tokens obtained from the corresponding original vectors $\boldsymbol{v}_{0}$ minus the center $V_{C}$ of the following four vectors: "database", "databases", "dataset", and "datasets". In other words, $\boldsymbol{v}=\boldsymbol{v}_{0}-\boldsymbol{V}_{C}$.

In these matrices, values of the similarity between dataset names are shown in the second quadrant and those between non-dataset names in the fourth quadrant. And a value of the similarity is expressed with a color, where the higher value of the similarity a cell has, the darker color the cell is filled i n. For example, a cell in the second quadrantshows the value of cosine similarity between a dataset name and another dataset name. 

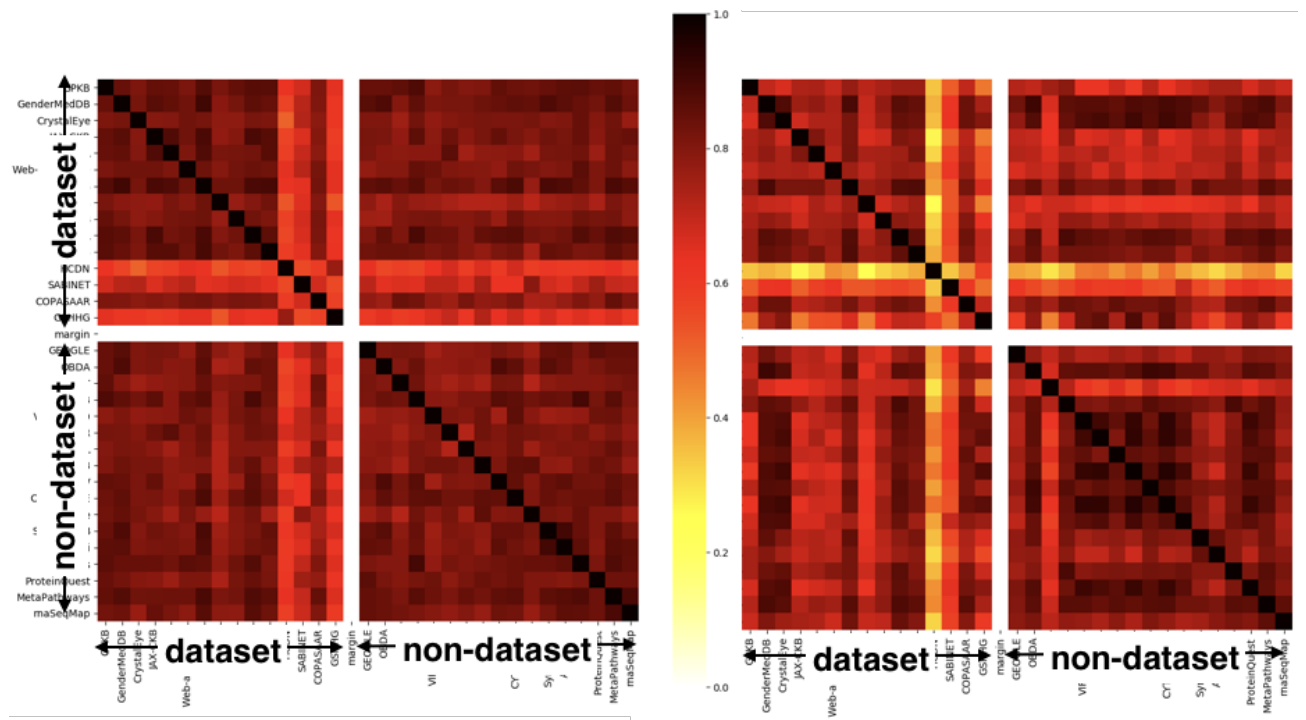

Figure 2: The left-hand (resp. right-hand) side figure shows values of cosine similarity between vectors $\boldsymbol{v}_{0}$ of tokens (resp. relative vectors $\boldsymbol{v}=\boldsymbol{v}_{0}-\boldsymbol{V}_{C}$, where pairs in the second quadrant are pairs of dataset names and those in the fourth quadrant those of non-dataset names, the higher value of the similarity a cell has, the darker color the cell is filled in, and $\boldsymbol{v}_{0}$ denotes an original vector of the trained word2vec model and $\boldsymbol{V}_{C}$ the center of the following four vectors: "database", "databases", "dataset", and "datasets".

In the left-hand figure, we see that the diagonal line is black since a cell on this line is a pair of the same token and thus these cells have highest similarity values. We also see some lines in second quadrant, that is, similarity values between some specific dataset name and other dataset names have relatively low values, colored in red.

In the right-handfigure of Figure 2, we find that values in the fourth quadrantare larger (darker) than those in the same quadrant of the left-hand side figure, meaning that nondataset names are located at similar directions from the center. We also find that values in the 2nd quadrant, pairs of dataset names, are lower than those in the same quadrant of the left-hand side figure, meaning that dataset names are located at different directions from the center. But, these facts do not mean that dataset names are far from the center non-dataset

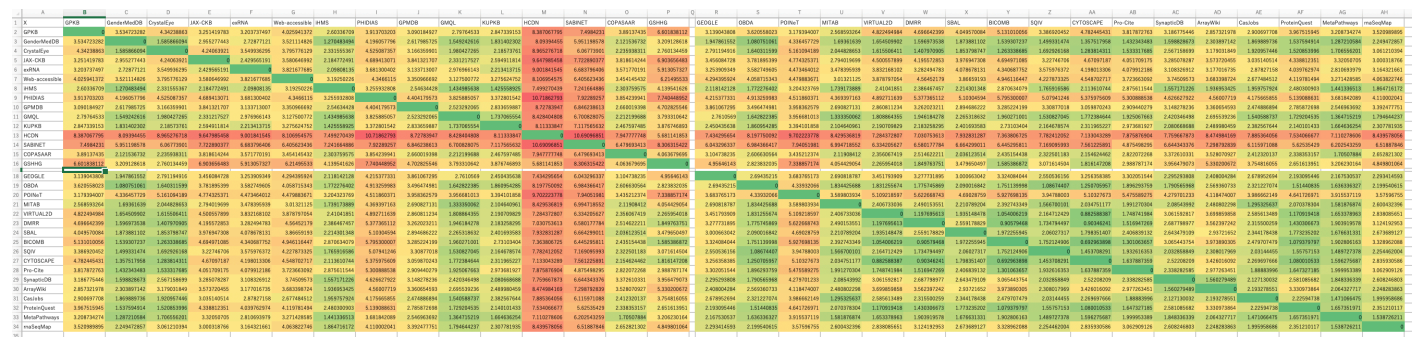

Figure 3: Distances between vectors of tokens, where the vector $v$ of a token is obtained from the original vector $\boldsymbol{v}_{0}$ of the trained word2vec model minus the center $\boldsymbol{V}_{C}$ of the following four vectors: "database", "databases", "dataset", and "datasets", that is, $\boldsymbol{v}=\boldsymbol{v}_{0}-$ $V_{C}$. 
names are close to the center because the cosine similarity value for two vectors shows an angle between them.

Figure 3 shows distances, not cosine similarity, between tokens and the center. In this figure, we see that the diagonal line is green since a cell on this line is a pair of the same token and so the distance is zero. From this figure, we also see that many cells in the fourth quadrantcolored in green, meaning non-dataset names are located close to each other. If we can identify these non-dataset names as one vector or in a simple notation, we can expect that they would be removed from the top output.

\section{Conclusion}

In this paper, the authors have proposed a method to identify dataset names in scholarly papers, and conducted experiments using real scholarly papers in many disciplines. Increasing the size of text data, the authors have improved the precision at top $\mathrm{N}$ list of outputs, from 0.35 to 0.45 . However, there are still many non-dataset names in top $\mathrm{N}$ list, it is an importantfuture work to remove these noise tokens.

\section{References}

[1] "NII Institutional Repositories Program | Documents | Statistics," https://www.nii.ac. jp/irp/en/archive/statistic/, accessed: 2020-03-04.

[2] "arXiv.org e-Print archive," https://arxiv.org/, accessed: 2020-03-04.

[3] "Home - PubMed - NCBI," https://www.ncbi.nlm.nih.gov/pubmed/, accessed: 202003-04.

[4] "RePEc: Research Papers in Economics," http://repec.olg/, accessed: 2020-03-04.

[5] "Home:: SSRN," https://www.ssrn.com/, accessed: 2020-03-04.

[6] D. Ikeda and P. Wang, "Revealing Presence of Amateurs at an Institutional Repository by Analyzing Queries at Search Engine," in Proceedings of the 7th International Conference of Open Repositories, 2012.

[7] Y. Ohira, K. Ogashiwa, S. Muranaga, T. Matsumoto, and H. Naitoh, "A Questionnaire System for Institutional Research,” Information Engineering Express, vol. 3, no. 1, pp. 9-18, 2017.

[8] K. Ogashiwa, T. Matsumoto, Y. Wang, J. Kariya, and H. Naitoh, "Evaluation of the Yamaguchi University Self-Assessment and Evaluation System and Its Improvement," International Journal of Institutional Research and Management, vol. 3, no. 1, pp. 1$14,2019$.

[9] D. Ikeda and D. Seguchi, "Automatically Extracting Keywords from Documents for Rich Indexes of Searchable Data Repositories," in Proceedings of the 12th International Conference of Open Repositories, 2017.

[10] B. Ghavimi, P. Mayr, S. Vahdati, and C. Lange, "Identifying and Improving Dataset References in Social Sciences Full Texts," ArXiv e-prints, 2016. 
[11] A. Singhal, R. Kasturi, and J. Srivastava, "DataGopher: Context-based Search for Research Datasets," in Proceedings of the 2014 IEEE 15th International Conference on Information Reuse and Integration, 2014, pp. 749-756.

[12] A. Singhal and J. Srivastava, "Data Extract: Mining Context from the Web for Dataset Extraction," International Journal of Machine Learning and Computing, vol. 3, no. 2, pp. 219-223, 2013.

[13] “CORE - Aggregating the world's open access research papers," https://core.ac.uk/, accessed: 2020-03-04.

[14] P. Knoth and Z. Zdrahal, "CORE: Three Access Levels to Underpin Open Access," D-Lib Magazine, vol. 18, no. 11/12, 2012.

[15] T. Mikolov, I. Sutskever, K. Chen, G. S. Corrado, and J. Dean, "Distributed Representations of Words and Phrases and their Compositionality," in Advances in Neural Information Processing Systems 26, C. Burges, L. Bottou, M. Welling, Z. Ghahramani, and K. Weinberger, Eds. Curran Associates, Inc., 2013, pp. 3111-3119.

[16] T. Mikolov, K. Chen, G. Corrado, and J. Dean, "Efficient Estimation of Word Representations in Vector Space," CoRR, 2013.

[17] C. D. Manning, P. Raghavan, and H. Schuetze, Introduction to Information Retrieval. Cambridge University Press, 2008.

[18] K. Järvelin and J. Kekäläinen, "Cumulated Gain-based Evaluation of IR Techniques," ACM Transactions on Information Systems, vol. 20, no. 4, pp. 422-446, 2002.

[19] D. Ikeda and Y. Taniguchi, "Toward Automatic Identification of Dataset Names in Scholarly Articles," in Developments in Open Science and Research Data Management: 8th International Conference on Data Science and Institutional Research, 2019.

[20] K. Gábor, D. Buscaldi, A.-K. Schumann, B. QasemiZadeh, H. Zargayouna, and T. Charnois, "SemEv al-2018 Task 7: Semantic Relation Extraction and Classification in Scientific Papers," in Proceedings of The 12th International Workshop on Semantic Evaluation. New Orleans, Louisiana: Association for Computational Linguistics, 2018, pp. 679-688. [Online]. Available: https://www.aclweb.org/anthology/S18-1111

[21] Y. Yamada, D. Ikeda, and S. Hirokawa, "Automatic Wrapper Generation for Multilingual Web Resources," in Proceedings of the 5th International Conference on Discovery Science, ser. Lecture Notes in Computer Science 2534. Springer-Verlag, 2002, pp. 332-339.

[22] D. Ikeda and Y. Yamada, "Gathering Text Files Generated from Templates," in Proceedings of Workshop on Information Integration on the Web (IIWeb-04), 2004, pp. $21-26$.

[23] R. L. Cilibrasi and P. M. Vitanyi, “The Google Similarity Distance," IEEE Transactions on Knowledge and Data Engineering, vol. 19, no. 3, pp. 370-383, 2007.

[24] A. Singhal and J. Srivastava, "Research Dataset Discovery from Research Publications Using Web Context," Web Intelligence, vol. 15, no. 2, pp. 81-99, 2017. 